\title{
Phenotype-driven strategies for exome prioritization of human Mendelian disease genes
}

\author{
Damian Smedley ${ }^{1}$ and Peter N. Robinson ${ }^{2,3,4,5^{*}}$ (D)
}

\begin{abstract}
Whole exome sequencing has altered the way in which rare diseases are diagnosed and disease genes identified. Hundreds of novel disease-associated genes have been characterized by whole exome sequencing in the past five years, yet the identification of diseasecausing mutations is often challenging because of the large number of rare variants that are being revealed. Gene prioritization aims to rank the most probable candidate genes towards the top of a list of potentially pathogenic variants. A promising new approach involves the computational comparison of the phenotypic abnormalities of the individual being investigated with those previously associated with human diseases or genetically modified model organisms. In this review, we compare and contrast the strengths and weaknesses of current phenotype-driven computational algorithms, including Phevor, Phen-Gen, eXtasy and two algorithms developed by our groups called PhenIX and Exomiser. Computational phenotype analysis can substantially improve the performance of exome analysis pipelines.
\end{abstract}

\section{Disease-associated gene discovery and genomic diagnostics}

It seems fair to say that next-generation sequencing (NGS)-based diagnostics are revolutionizing the way that rare diseases are diagnosed and researched. For example, programs such as Care4Rare [1], the program at the Centers for Mendelian Genomics [2], and the Undiagnosed Diseases Program of the National Institutes for Health [3] have developed computational and clinical frameworks for the efficient identification of novel genes

\footnotetext{
* Correspondence: peter.robinson@charite.de

${ }^{2}$ Institute for Medical Genetics and Human Genetics,

Charité-Universitätsmedizin Berlin, Berlin, Germany

${ }^{3}$ Max Planck Institute for Molecular Genetics, Ihnestrasse, 14195 Berlin, Germany

Full list of author information is available at the end of the article
}

implicated in disease. Furthermore, clinical groups have shown the utility of exome and genome sequencing in improving the diagnosis of rare genetic diseases [4-11]. The UK 100,000 Genomes Project, which aims to transform the way that genomics is used in the National Health Service (NHS), is focused on the areas of rare disease, infectious disease and cancer. This project has recently reported the first successful diagnoses of patients using exome sequencing [12] (Box 1). Detailed clinical phenotyping is a keystone of the UK 100,000 Genomes Project's strategy; the aim is to use phenotypic analysis to guide the interpretation of genome sequence data that cover at least $95 \%$ of the genome at 15 -fold or better.

Many clinical centers are now using whole exome sequencing (WES). This process relies on oligonucleotide probes to capture (hybridize to) the target exonic sequences from fragmented total genomic DNA, followed by enrichment and NGS of the targeted sequences [13]. WES is typically performed using kits that aim to capture all exonic and flanking sequences and may also include probes to target microRNA and other sequences of interest [14]. Recent large-scale clinical WES studies have reported a successful molecular diagnosis in up to $25 \%$ of cases in large cohorts of unselected, consecutive patients $[6-8,15]$. Despite this progress, it remains difficult to identify causative mutations in the genomes of many patients.

A number of strategies have emerged to rank the variants and the genes that they affect, with those most likely to cause disease ranked highest, through a process termed gene prioritization [16-18]. Current approaches towards gene prioritization include simultaneously sequencing multiple affected individuals and searching for genes that are affected in all or most individuals [17], linkage analysis [19], and various forms of network analysis [20]. The first two strategies identify specific genes or genomic intervals as candidates, whereas network approaches generate a relative likelihood that every gene in the genome is causal. An additional strategy that is 
Box 1. Prominent exome sequencing projects in the field of rare disease research

A number of large-scale, multicenter projects have emerged in recent years that aim to use whole exome sequencing (WES) to discover novel disease-associated genes and to improve the diagnosis and treatment of rare hereditary diseases. These include:

- Care4Rare (http://care4rare.ca/). This project has emerged from the Canadian FORGE (Finding of Rare Disease Genes) initiative, which has been able to identify disease-causing variants for 146 of the 264 disorders studied over a 2-year period, with up to 67 novel disease-associated genes being characterized [63].

- Centers for Mendelian Genomics (CMG) (http://www. mendelian.org/). A group of sequencing centers funded by the National Institutes of Health has established three CMGs (Baylor-Johns Hopkins CMG, the University of Washington CMG and the Yale CMG) [64].

- Undiagnosed Disease Program of the National Institutes of Health (http://www.genome.gov/27550959). The

Undiagnosed Disease Program was founded with the goal of achieving a diagnosis for patients who remained undiagnosed after an exhaustive workup and to discover new disorders that would provide insight into mechanisms of disease [65].

- The UK 100,000 Genomes Project (http://www.genomics england.co.uk/). This project includes a major focus on rare inherited diseases with the goal of introducing genomics diagnostics into the mainstream healthcare system for the benefit of patients and researchers.

\section{- DECIPHER (DatabasE of genomiC varlation and} Phenotype in Humans using Ensembl Resources) (https://decipher.sanger.ac.uk/). This resource has been in operation since 2004 and represents a community driven database of array comparative genomic hybridization (CGH) and WES data that can be used for genomic matchmaking [66].

- The Deciphering Developmental Disorders (DDD) study (http://www.ddduk.org/) has the goal of improving diagnostics of developmental disorders in children by means of array CGH and next-generation sequencing methods. The program has achieved a diagnostic yield of $27 \%$ among 1133 previously investigated yet undiagnosed children who have developmental disorders [67].

- The Global Alliance for Genomics and Health coordinates several groups that are involved in genomic matchmaking, which allows physicians to search for patients with similar genotypes and phenotypes to facilitate and accelerate novel disease-associated gene discovery. Many of these databases, such as PhenomeCentral (https://phenomecentral.org/), use phenotype analysis.

The analysis of data in these and other projects benefits greatly from other collections of exome data that allow the frequency of variants in the population to be estimated (for instance, in order to filter out variants whose population frequency exceeds a certain threshold). These include the NHLBI-ESP 6500 exome project (https://esp.gs.washington.edu/drupal/), the Exome Aggregation Consortium (ExAC) (http://exac.broadinstitute.org/), and the 1000 Genomes Project [23].

proving particularly successful uses knowledge of the patient's phenotype to assess candidate sequences.

In this review, we provide an overview of the current tools that use computational analysis of the phenotype as a major component of their exome prioritization procedures. We explain how phenotype-driven analysis of exome data can be used to filter out common variants and those deemed to be non-pathogenic. We also present a number of recently published tools that substantially improve the analysis of WES data by incorporating phenotypic features into their prioritization procedures, and compare their strengths and weaknesses.

\section{Variant annotation and filtering}

Exome analysis of the tens of thousands of sequence variants typically found in any individual usually begins with filtering out of target and high-frequency variants. In many cases, the remaining variants are filtered or prioritized on the basis of their predicted pathogenicity. An essential step in the interpretation of these data is the annotation of these variants with respect to their potential effects on genes and transcripts; this requires the translation of variant-describing semantics in the Variant Call Format (VCF), which reflects the chromosomal coordinates of each variant (for example, chr10:g.123256215T>G), into gene-based variant annotations (such as c.518A $>$ C; p.Glu173Ala in the gene FGFR2). This is necessary because evaluation of a variant in a diagnostic context almost always requires assessment of the potential effects of variants on gene products [21].

Several annotation tools offer additional functionality that allows variants to be filtered according to their population frequency and variant class. For instance, ANNOVAR [22] annotates variants relative to a number of popular gene sets to identify the functional consequence of the mutation; for example, new amino acid (missense) or stop-codon (nonsense) mutations can result from a non-synonymous point mutation. In addition, this tool can filter variants to produce a more manageable set 
of candidates on the basis of various criteria, such as excluding any common single nucleotide polymorphisms (SNPs) present in dbSNP or present with a minor allele frequency (MAF) more than $1 \%$ in the 1000 Genomes Project [23] or NHLBI-ESP 6500 exome project (ESP) datasets. Other sources of data that can be used for prioritization include deleteriousness scores precomputed using the variant-analysis tools Sorting Intolerant from Tolerant (SIFT) [24], Polymorphism Phenotyping (PolyPhen) [25], Genomic Evolutionary Rate Profiling (GERP) [26], and Combined Annotation-Dependent Depletion (CADD) [27] (Box 2). Finally, the exome annotation tool Jannovar can implement the expected inheritance model

\section{Box 2. Selection of tools used for the analysis of variants found in whole exome sequencing data}

Variant annotation tools translate the genomic coordinates of

variants given by variant call format (VCF) files (which are

commonly used in exome sequencing) into the corresponding

transcript-based annotations. ANNOVAR annotates variants in this

way and performs tasks such as examining their functional

consequence on genes. In addition, this tool performs functional

annotation of the variants with respect to a number of attributes

[22]. Jannovar performs such annotation as well as pedigree-based

analysis and can also be used as a Java programming library [21].

Pathogenicity prediction programs use computational

analysis to assess the potential impact of amino acid

substitutions, and in some cases other categories of variants, on

protein function. Sorting Intolerant from Tolerant (SIFT) uses

sequence homology to predict the likelihood that an amino

acid substitution will have an adverse effect on protein function

[68]. Polymorphism Phenotyping v2 (PolyPhen-2) predicts the

impact of amino acid substitutions on the stability and function

of affected proteins using structural and comparative

evolutionary comparisons [25]. MutationTaster uses Bayesian

methodologies to predict the relevance of a wide range of

variants [69]. The Combined Annotation scoRing toOL (CAROL)

combines the predictions of PolyPhen-2 and SIFT [70]. The

Combined Annotation-Dependent Depletion (CADD) integrates

a large number of sequence and genomic attributes to train a

support vector machine to predict deleteriousness [27]. Genomic

Evolutionary Rate Profiling (GERP) is a method to assess regions

that have been subject to purifying selection and are enriched

for functional elements [26].

Variant annotation pathogenicity prediction tools are used to

assess the potential relevance of variants in WES data. In

phenotype-driven exome analysis, the final ranking of the genes

that contain these variants is performed using phenotypic analysis

according to the algorithms described for the several programs. for further filtering [21]. The Variant Effect Predictor [28] of the European Bioinformatics Institute (EBI) can be used through either an online interface, a downloadable Perl command-line tool or a scalable web service such as RESTful. Variants can be input in a number of formats (VCF, Human Genome Variation Society (HGVS) and so on) and the functional consequence annotated using a number of transcript sets (Ensembl, Gencode or Refseq). Filters can be set to exclude non-coding variants or common variants above a certain MAF in the variant populations provided by the1000 Genomes Project [23], the Exome Sequencing Project [29], or the Exome Aggregation Consortium [30]. The output also includes predicted deleteriousness scores from SIFT and PolyPhen.

\section{Phenotype-based exome analysis tools}

When the diagnosis is not known in advance, or if a novel disease gene is being sought, computational phenotype analysis can serve to assess each candidate gene's relevance to the clinical abnormalities observed in the patient(s). Although other ontologies or terminologies that represent phenotypes exist (such as SNOMED CT, MeDRA, London Dysmorphology Database, POSSUM, PhenoDB, ICD-9/10/11) [31] the current applications in this field make use of the Human Phenotype Ontology (HPO) database, which aims to provide a computable representation of the clinical abnormalities observed in human disease [32]. A number of algorithms have been developed to estimate the similarity between two diseases based on their phenotypic features encoded using HPO terms [33]. These algorithms can be adapted to measure the similarity between a set of query terms representing the clinical manifestations observed in a patient and those representing each of the diseases in a database [34-37]. The algorithms below utilize an assessment of clinical similarity to prioritize candidate genes.

\section{eXtasy}

eXtasy [38] takes a data integration approach (genomic data fusion [39]) to variant prioritization. To generate an overall prediction of causality, ten different measures of variant deleteriousness that are available from existing tools and databases, along with a gene haploinsufficiency prediction score, are combined with a phenotype-specific gene score. The phenotype-based method takes all disease genes known to be associated with a particular HPO term or terms from Phenomizer [37] and scores the similarity of each candidate gene in the exome to this gene set using the Endeavour algorithm [39]. Endeavour uses various measures of gene similarity, such as sequence similarity and co-expression, as well as involvement in the same protein-protein interactions or pathways. A Random Forest algorithm is used to produce a single combined candidacy score from all of these sources of evidence. For 
variants that are missing data from any of the methods, an imputed score is calculated that ignores haploinsufficiency and uses median values across all variants for the missing deleteriousness scores.

Receiver operating characteristic (ROC) analysis was used to assess the ability of eXtasy to discriminate diseasecausing from rare control variants or common polymorphisms. This analysis showed substantial improvement when compared with classical deleterious prediction methods such as PolyPhen, SIFT, MutationTaster and CAROL. Currently, eXtasy only performs prioritization of non-synonymous variants but when public datasets that are sufficiently large for training become available, it will be expanded to include mitochondrial, noncoding, synonymous and nonsense variants, as well as mutations around the splice junction that affect splicing and insertion and deletion of base mutations (indels). eXtasy performs no filtering, so it is recommended that the exome is pre-filtered to remove off-target or common (MAF $>1 \%$ ) variants. eXtasy is available for online use or download [40].

\section{Phevor: Phenotype Driven Variant Ontological Re-ranking tool}

Phevor [41] takes the outputs of variant-prioritization tools such as ANNOVAR or the Variant Annotation, Analysis, Search Tool (VAAST) [42] and then prioritizes the remaining genes using phenotype, gene function and disease data. This knowledge comes from publically available gene annotation sets using various biomedical ontologies such as the HPO, Mammalian Phenotype Ontology (MPO) [43, 44], Disease Ontology (DO) [45], and Gene Ontology (GO) [46]. Users specify a list of terms from one or more of HPO, DO, MPO, GO or Online Inheritance in Man (OMIM) [47] that characterize what is known about the patient. Phevor then generates a list from genes that have been annotated with these terms or their parent terms if no gene annotations exist. Next, it identifies terms in the other ontologies that are annotated to these genes and the process is repeated to expand the gene list. Thus, concepts in different ontologies are related through their annotation of the same gene. Finally, each gene receives a score based on propagation from the seed nodes in each ontology and a combination procedure across the scores from the various ontologies. The final Phevor score combines the ranking information for the variant prioritization tool (or $P$-value from VAAST) with this gene score.

Benchmarking of Phevor on simulated disease exomes, based on in-house generated exomes, demonstrated a considerable improvement over variant prioritization methods such as ANNOVAR and VAAST, with 95-100\% of the exomes having the causative variant in the top ten candidates. Three case studies where Phevor was used to identify disease-causing alleles have also been presented. Phevor is available for online use only [48].

\section{Phen-Gen}

Phen-Gen [49] uses a Bayesian framework to compare predicted deleterious variants in the patient's exome and known patient symptoms to prior knowledge of human disease-gene associations and gene interactions. Coding variants are analyzed using a unifying framework to predict the damaging impact of non-synonymous, splicesite and indel variants. Phen-Gen also allows a genomewide approach in which evolutionary conservation and Encyclopedia of DNA Elements (ENCODE)-predicted functionality and proximity to coding sequences are used to score non-coding variants.

Any variant that has a MAF above $1 \%$ is removed from further analysis. Healthy individuals contain many damaging mutations and the fact that this ability to tolerate mutations varies from gene to gene is also taken into account using a null model. This model uses the observed variants from the 1000 Genomes Project to generate a null distribution under either a dominant or recessive inheritance model for each gene. Genes are only retained for further analysis if the predicted damaging score for the variants exceeds that seen for $99 \%$ of the 1000 Genomes dataset.

These remaining genes are then analyzed using the Phenomizer algorithm to match semantically the patient's phenotypes encoded using HPO to known disease-gene associations. The role of novel (non-disease genes) is assessed by identifying functionally related genes using a random-walk-with-restart algorithm over a gene interaction network. Phenotype matches are distributed to these novel genes across the network such that the disease gene hub gets the majority (90\%) of the score and other genes get a share of the remainder, according to their proximity to the disease gene.

Benchmarking using simulated exomes that were based on 1000 Genomes Project data showed that the correct disease variant was obtained as the top hit in $88 \%$ of samples. Using a strategy in which known associations were masked to simulate the discovery of novel associations, performance figures of $56 \%$ and $89 \%$ were obtained for dominant and recessive disorders, respectively. In an evaluation using real patient data, 11 trios with recessive or X-linked intellectual disability were analyzed and $81 \%$ of the reported genes were in the top ten candidates. Phen-Gen is available for online use or download [49].

\section{Exomiser}

The original implementation of Exomiser [50] used semantic similarity comparisons between patient phenotypes 
and mouse phenotype data for each candidate gene in the exome. The PhenoDigm [51] algorithm is used to score each gene from 0 to 1 , where 1 represents the perfect match and genes with no data received a default score of 0.6 . This phenotype score is combined with a variant score that is based on the allele rarity in the 1000 Genomes Project and ESP datasets together with predictions of deleteriousness from PolyPhen, SIFT and MutationTaster.

Benchmarking on simulated exomes based on 1000 Genomes Project data showed that $66 \%$ of cases had the causative variant as the top hit under a dominant model and $83 \%$ under a recessive model [50].

Exomiser has been improved subsequently to include comparison with human and fish phenotypes, as well as use of a random-walk with restart to score genes with no phenotype data (genes are scored based on proximity in the StringDB interaction network to other genes that do show phenotypic similarity to the patient data) [20]. Exomiser is available as an online web service [52] or for download as a command-line tool. Installation simply involves unzipping the download.

\section{PhenIX}

PhenIX [5] uses the same software framework as Exomiser but instead of using human, mouse, fish, and proteinprotein association data, this tool is restricted to comparisons between patient phenotypes and known disease gene phenotypes. This simplification is made because PhenIX is intended for diagnostic tasks when only known disease genes can be reported. In addition, the semantic similarity algorithm uses the Phenomizer algorithm [37].

Benchmarking on sequence files generated from a target enrichment panel that was based on known diseaseassociated genes revealed that $97 \%$ of samples had the inserted variant as the top hit, regardless of inheritance model. The same performance was observed when using 1000 Genomes Project exomes.

PhenIX is available in the same downloadable library as Exomiser and has the same filtering options. In addition it can be used from its website [52].

\section{Comparison of exome prioritization tools}

Table 1 summarizes the main features of the software solutions described above. For clinicians and many researchers, a well-designed web interface solution is best in terms of usability. Installation of the command-line versions of the tools will be difficult or off-putting for many such users. Nevertheless, web-based solutions present security issues in that patient exomes have to be uploaded onto external servers. To counter this, publically available, secure, cloud-based versions or easy-to-install local clients would be welcomed in the future. By contrast, for many medium-to-large projects, the primary users of these tools are going to be the bioinformatics teams that support clinical researchers. For these users, a commandline version that can be integrated into their pipelines is the most useful platform; for example, some of the tools can take as input VCF files from one program and can output VCF that can feed into another.

To further compare these tools, benchmarking was performed on 50 simulated disease exomes, generated by randomly adding known non-synonymous disease variants (two copies for recessive diseases and one for dominant) from the Human Genome Mutation Database (HGMD) to either 50 randomly chosen unaffected exomes from the 1000 Genomes Project or 50 exomes generated by us in-house (Fig. 1). The diseases and variants used for the benchmarking of the 50 exomes in Fig. 1 are detailed in Additional file 1. Two background sources of exome data were used because the 1000 Genomes Project exomes can over-predict the performance that will be obtained for real patient exomes. This is because many of the tools utilize the allele frequency data from the 1000 Genomes Project for filtering and prioritization. Data from the 1000 Genomes Project variants have also been used to train some of the algorithms. In addition, real patient exomes typically contain many more variants than the conservatively called 1000 Genomes Project exomes; for example, our in-house generated exomes contain $140,000-231,000$ variants compared to $24,000-42,000$ in the 1000 Genomes Project exomes.

Exomiser and PhenIX were run from the commandline with the default settings and MAF filter set to $<1 \%$ and the appropriate inheritance model specified. PhenGen was run from the command line, again with the inheritance model specified. EXtasy was run from the command line using just the phenotypes as additional arguments. EXtasy does not perform any variant filtering, so to allow a better comparison with the other tools, we ran it on the filtered variants from Exomiser. Phevor is also just a variant prioritizer and relies on a filtered exome from software such as VAAST or ANNOVAR. Hence, we used the output of ANNOVAR's variant_ reduction.pl script with the default settings along with specification of the inheritance model. Table 2 shows the average gene counts before and after filtering by these various strategies.

HPO annotations for the disease under consideration were included in the prioritization analysis for each software. We assessed performance when using: (a) all available phenotypes, (b) a maximum of three phenotypes randomly chosen from the annotations, (c) the same three phenotypes but with two promoted to the lessspecific parent term and two false-positive terms randomly chosen from the whole of HPO. Phevor only allows up to five HPO terms, so only the latter two options were tested for this tool. 
Table 1 Comparison of exome analysis tools

\begin{tabular}{|c|c|c|c|c|}
\hline Software & Exome input & Types of variant analyzed & Availability & Software approach \\
\hline VEP & $\begin{array}{l}\text { Various including VCF, } \\
\text { pileup, HGVS notations }\end{array}$ & All & $\begin{array}{l}\text { Website, } \\
\text { command line } \\
\text { and REST service }\end{array}$ & $\begin{array}{l}\text { Filtering by allele frequency and deleteriousness scores } \\
\text { (SIFT, PolyPhen) }\end{array}$ \\
\hline ANNOVAR & $\begin{array}{l}\text { Various including } \\
\text { multi-sample VCF }\end{array}$ & All & Command line & $\begin{array}{l}\text { Filtering by allele frequency, inheritance model and } \\
\text { deleteriousness scores (SIFT, PolyPhen, MutationTaster, } \\
\text { MutationAssessor, LRT, FATHMM, MetaSVM, MetaLR, } \\
\text { GERP++, PhyloP, SiPhy, CADD) }\end{array}$ \\
\hline eXtasy & Single sample VCF & Non-synonymousonly & $\begin{array}{l}\text { Website and } \\
\text { command line }\end{array}$ & $\begin{array}{l}\text { Prioritization based on a Random Forest score from } \\
\text { combined deleteriousness scores (CAROL, LRT, } \\
\text { MutationTaster, PhastCons, PhyloP, PolyPhen, SIFT), } \\
\text { haploinsufficiency, and similarity of the gene to genes } \\
\text { annotated with the input Human Phenotype Ontology } \\
\text { (HPO) phenotypes as measured by sequence similarity, } \\
\text { co-expression, and involvement in the same pathway } \\
\text { or protein-protein interactions }\end{array}$ \\
\hline Phevor & $\begin{array}{l}\text { Pre-filtered VAAST or } \\
\text { ANNOVAR files or } \\
\text { functionally annotated } \\
\text { multi-sample VCF }\end{array}$ & All & Website & $\begin{array}{l}\text { Prioritization based on semantic similarity of each } \\
\text { candidate gene to genes annotated with the input } \\
\text { set of ontology terms taken from HPO, Mammalian } \\
\text { Phenotype Ontology (MPO), Disease Ontology (DO), } \\
\text { and Gene Ontology (GO) }\end{array}$ \\
\hline Phen-Gen & $\begin{array}{l}\text { Multi-sample } \\
\text { family VCF }\end{array}$ & All & $\begin{array}{l}\text { Website and } \\
\text { command line }\end{array}$ & $\begin{array}{l}\text { Filtering by inheritance model and stringency } \\
\text { or reentrance. } \\
\text { Prioritization based on predicted variant impact and } \\
\text { semantic phenotypic similarity between HPO input and } \\
\text { HPO-annotated diseases associated with each exomic } \\
\text { candidate or its neighbors in an interaction network }\end{array}$ \\
\hline PhenIX & $\begin{array}{l}\text { Multi-sample } \\
\text { family VCF }\end{array}$ & All coding & $\begin{array}{l}\text { Website and } \\
\text { command line }\end{array}$ & $\begin{array}{l}\text { Filtering by allele frequency, variant quality, and } \\
\text { inheritance model. } \\
\text { Prioritization based on predicted deleteriousness } \\
\text { (SIFT, PolyPhen, MutationTaster), allele frequency and } \\
\text { semantic phenotypic similarity between HPO input } \\
\text { and HPO-annotated diseases associated with each } \\
\text { exomic candidate }\end{array}$ \\
\hline Exomiser & $\begin{array}{l}\text { Multi-sample } \\
\text { family VCF }\end{array}$ & All coding & $\begin{array}{l}\text { Website and } \\
\text { command line }\end{array}$ & $\begin{array}{l}\text { Filtering by allele frequency, variant quality, } \\
\text { deleteriousness scores and inheritance model. } \\
\text { Prioritization based on predicted deleteriousness } \\
\text { (SIFT, PolyPhen, MutationTaster), allele frequency and } \\
\text { semantic phenotypic similarity between HPO input and } \\
\text { HPO-annotated diseases, MPO-annotated mouse and } \\
\text { Zebrafish Phenotype Ontology (ZPO)-annotated fish } \\
\text { models associated with each exomic candidate or its } \\
\text { neighbors in an interaction network }\end{array}$ \\
\hline
\end{tabular}

Abbreviations: CADD Combined Annotation-Dependent Depletion, GERP Genomic Evolutionary Rate Profiling, HGVS Human Genome Variation Society, HPO Human Phenotype Ontology, LRT likelihood ratio test (LRT), PolyPhen Polymorphism Phenotyping, REST Representational State Transfer, SIFT Sorting Intolerant from Tolerant, VAAST Variant Annotation, Analysis, Search Tool, VCF variant call format

Fifty exomes is too small a number to make statistically valid conclusions on the performance of each tool, but we were limited to this number as we wanted to include Phevor and this was only available through manual, web use. However, the results from 1000 exomes run through the other tools (Fig. 2) did not differ much from that seen from 50 exomes, so the results are likely to be representative. In addition, the results are in rough agreement with previously published reports of performance using a similar strategy: $97 \%$ as the top hit using PhenIX or Exomiser, $88 \%$ as the top hit with Phen-Gen, and $95 \%$ in the top 10 for Annovar plus Phevor.
As expected, the tools that took advantage of phenotype data outperformed prioritization tools that rely on variant analysis alone. For the exomes that were based on the 1000 Genomes Project, Exomiser, PhenIX, PhenGen and Phevor clearly outperformed eXtasy, with PhenIX looking like the best option when the phenotype is clearly defined and Exomiser performing the best when missing, generalized and atypical phenotypes are present. The same pattern was seen for the analyses of the samples based on our in-house-generated exomes, except that the performance of Phen-Gen decreased dramatically such that it was the worst performing tool. Phen-Gen was 


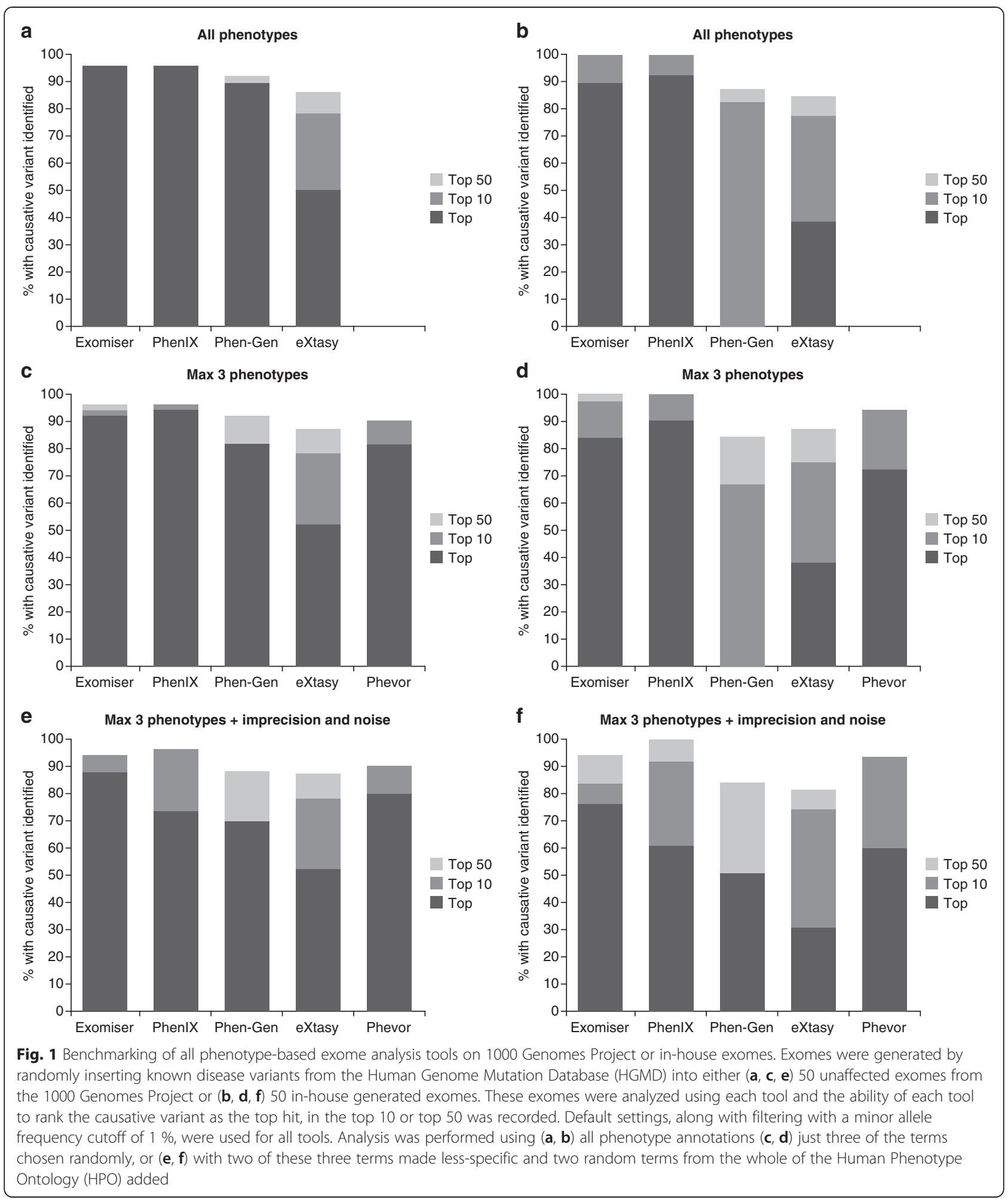

unable to prioritize any of the disease variants as the top hit in these samples. Phen-Gen uses a measure of genic intolerance that is based on 1000 Genomes Project data, and it could be that this plays a large part in the impressive performance of this tool when analyzing the simulated 1000 Genomes-based exomes. As shown in Table 2, the dramatic filtering Phen-Gen achieved when working with the 1000 Genomes Project-based exomes 
Table 2 Number of genes per benchmarked sample

\begin{tabular}{|c|c|c|c|c|}
\hline & \multicolumn{2}{|c|}{1000 Genomes Project exomes } & \multicolumn{2}{|c|}{ In-house exomes } \\
\hline & $\mathrm{AD}$ & $A R$ & $\mathrm{AD}$ & $A R$ \\
\hline Before filtering & $10542 \pm 783$ & $10631 \pm 802$ & $19235 \pm 916$ & $19712 \pm 976$ \\
\hline Exomiser filtered & $388 \pm 110$ & $38 \pm 11$ & $973 \pm 104$ & $557 \pm 74$ \\
\hline PhenIX filtered & $388 \pm 110$ & $38 \pm 11$ & $973 \pm 104$ & $557 \pm 74$ \\
\hline Exomiser filtered for extasy analysis & $388 \pm 110$ & $38 \pm 11$ & $973 \pm 104$ & $557 \pm 74$ \\
\hline Phen-Gen filtered & $100 \pm 34$ & $5 \pm 4$ & $665 \pm-86$ & $331 \pm 70$ \\
\hline Annovar filtered for Phevor analysis & $88 \pm 36$ & $2 \pm 1$ & $372 \pm 61$ & $52 \pm 17$ \\
\hline
\end{tabular}

Abbreviations: $A D$ autosomal dominant, $A R$ autosomal recessive

was not reproduced for our in-house exomes. This is likely to be primarily related to the fact that frequency data are available for all variants in the 1000 Genomes Project exomes, but in-house data are likely to have 5-10 $\%$ 'private' variants with no available frequency data.

In terms of ease of use for the benchmarking, the tools that were available for download and command-line usage were clearly more convenient and suitable for high-throughput analysis. Exomiser, PhenIX and Annovar took 1-2 minutes to run each sample, but Phen-Gen took around 20 minutes and EXtasy took up to 50 minutes. When running on the Annovar pre-filtered results, Phevor takes less than a minute but a lot of initial manual work must be performed to generate the ANNOVAR file, upload it, enter all the HPO terms and launch the analysis.

\section{Outlook: the future of phenotypic-driven analysis of genomic data}

In this review, we have examined contemporary phenotypedriven exome analysis software. We performed an evaluation of several contemporary programs. Although the performance of the programs in tests such as ours is likely to depend on the way testing is performed, our results give a general idea of the performance that may be expected from phenotype-driven analysis of exomes in real experiments. We note, however, that not all individuals undergoing exome sequencing to evaluate a suspected rare disease will have a mutation that can be detected by exome sequencing; for instance, some patients with Mendelian disease may have mutations in distal enhancer sequences [53]. Every simulated patient in our analysis had a mutation that was detectable by exome sequencing, and so the rate of identification of causal mutations by phenotype-driven analysis of real exome data may be lower than that in our simulations. In addition, all of the tools we examined, with the exception of Phen-Gen, are likely to be systematically biased by training on known disease variants, which are almost always in coding regions. Finally, we suggest that the performance of phenotype-driven exome analysis software would be improved by better and more detailed phenotypic annotations [54]. Even with these limitations, however, the performance of programs such as Phevor, eXtasy, PhenGen, PhenIX, and Exomiser [5, 38, 41, 49, 50, 55] has clearly demonstrated the value of computational phenotype analysis for the interpretation of exome sequencing data from individuals with rare genetic disease.

While large-scale phenotyping initiatives have become almost routine for model organisms such as the mouse [56], rat [57, 58], and zebrafish [59], similar large-scale efforts for human disease have been lacking. The HPO project [32] and the Monarch Initiative [60] are developing resources towards providing a sound foundation for the annotation and computational analysis of phenotypic abnormalities in human disease and model organisms. A spate of challenges and opportunities remain: for example, improved ontological resources and more detailed annotations are required, especially for conditions such as behavioral abnormalities [33] and for 'new' phenotypes that are observable only with recently introduced technologies, such as abnormalities found upon glycomics analysis or muscle anomalies detectable by magnetic resonance imaging. More detailed phenotyping of larger cohorts of patients together with mutation data may help us to understand genotype-phenotype correlations. In this sense, it is important that the Leiden Open Variation Database (LOVD) software is increasingly capturing phenotype data on individual mutations, and offers the ability to use HPO terms [61].

One of the major goals of computational phenotype analysis of the kind described here is to empower the analysis of NGS data, not only in the context of rare disease but also in the context of personalized medicine. One of the goals of personalized medicine is to classify patients into subpopulations that differ with respect to disease susceptibility, phenotypic or molecular subclass of a disease, or the likelihood of a positive or adverse response to a specific therapy. The related concept of 'precision medicine', whose goal is to provide the best 


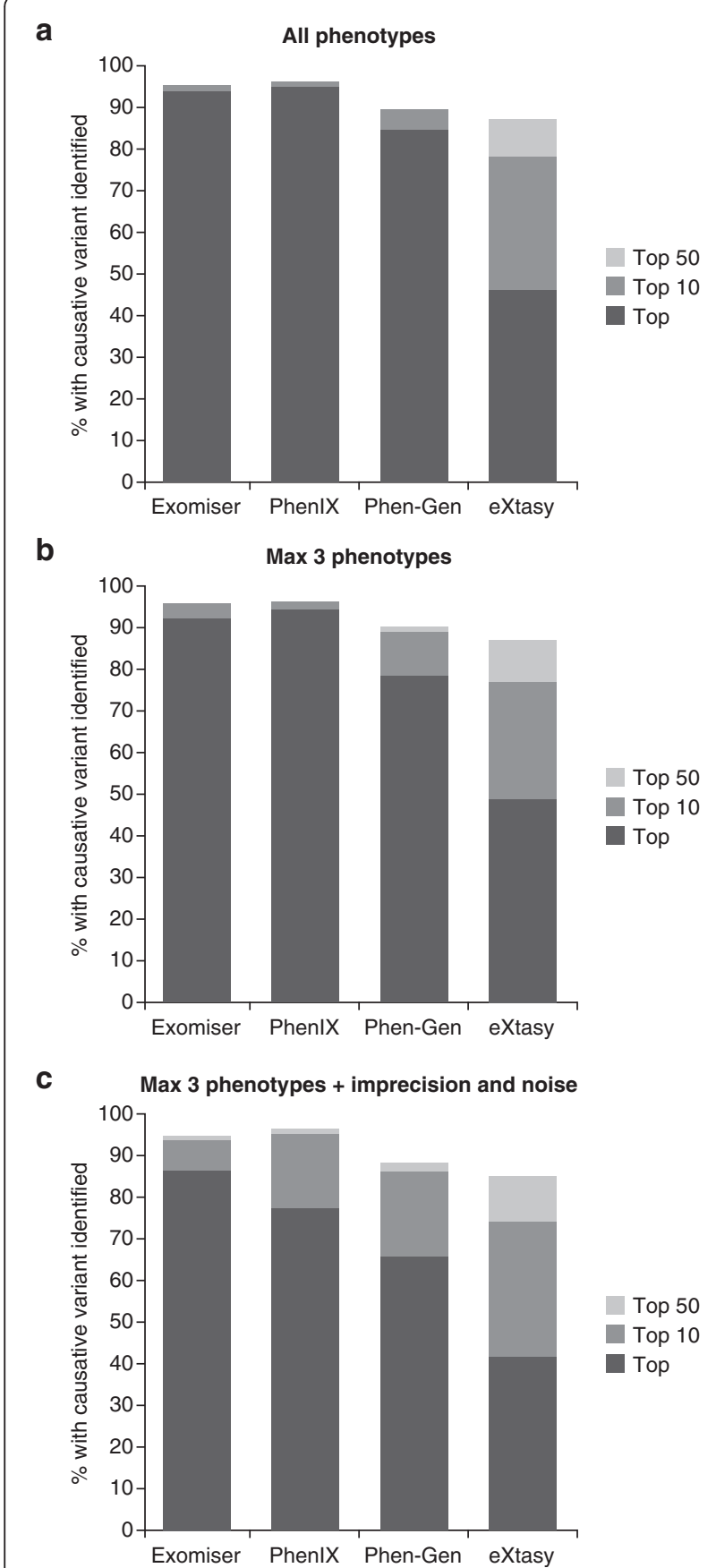

Fig. 2 Benchmarking of command-line exome analysis software. Exomes were generated by randomly inserting known disease variants from the Human Genome Mutation Database (HGMD) into 1000 unaffected exomes from the 1000 Genomes Project. These were analyzed using each tool and the ability of each to rank the causative variant as the top hit, in the top 10 or top 50 was recorded. Default settings along with a minor allele frequency cutoff of $1 \%$ were used for all. Analysis was performed using all phenotype annotations (a), just three of the terms chosen randomly (b), or with two of these three terms made less-specific and two random terms from the whole of the Human Phenotype Ontology (HPO) added (c) available care for each individual, refers to the stratification of patients into subsets each with a common biological basis of disease, such that stratified medical management is most likely to benefit the patients [62]. All medically relevant disease sub-classifications can be said to have a distinct phenotype, with the understanding that a medical phenotype comprises not only the abnormalities described but also the response of a patient to a certain type of treatment (for example, responsiveness of seizures to valproic acid can be considered to be a phenotype of certain forms of epilepsy). Therefore, comprehensive and precise phenotypic data, combined with ever increasing amounts of genomic data, appear to have an enormous potential to accelerate the identification of clinically actionable complications and of disease subtypes with prognostic or therapeutic implications.

The algorithms presented in this review probably represent only the first generation of increasingly powerful computational tools that will combine phenotype analysis and the investigation of genetic variants identified by WES or whole genome sequencing with the study of human disease and the practice of medicine.

\section{Additional file}

Additional file 1: Table S1. Detailing the diseases and variants used for the benchmarking of the 50 exomes in Fig. 1.

\section{Abbreviations}

CADD: Combined Annotation-Dependent Depletion; CAROL: Combined Annotation scoRing toOL; CGH: comparative genomic hybridization; DO: Disease Ontology; CMG: Center for Mendelian Genomics; ESP: NHLBI-ESP 6500 exome project; GERP: Genomic Evolutionary Rate Profiling; GO: Gene Ontology; HGMD: Human Genome Mutation Database; HPO: Human Phenotype Ontology; MAF: minor allele frequency; MPO: Mammalian Phenotype Ontology; NGS: next-generation sequencing; Phevor: Phenotype Driven Variant Ontological Re-ranking tool; PolyPhen: Polymorphism Phenotyping; SIFT: Sorting Intolerant from Tolerant; VAAST: Variant Annotation, Analysis, Search Tool; VCF: variant call format; WES: whole exome sequencing.

\section{Competing interests}

Peter Robinson reports patents US20110040766 A1 and WO2011018245 A1 involving methods for searching using ontologies.

\section{Acknowledgements}

The authors would like to acknowledge their many colleagues in their groups and at the Monarch Initiative (http://monarchinitiative.org/). This work was supported by grants from the Bundesministerium für Bildung und Forschung (BMBF 0313911) and from the European Commission's Seventh Framework Program (SYBIL, 602300) and by core infrastructure funding from the Wellcome Trust.

\section{Author details}

${ }^{1}$ Skarnes Faculty Group, Wellcome Trust Sanger Institute, Hinxton, UK. ${ }^{2}$ Institute for Medical Genetics and Human Genetics, Charité-Universitätsmedizin Berlin, Berlin, Germany. ${ }^{3}$ Max Planck Institute for Molecular Genetics, Ihnestrasse, 14195 Berlin, Germany. ${ }^{4}$ Berlin Brandenburg Center for Regenerative Therapies (BCRT), Charité-Universitätsmedizin Berlin, Augustenburger Platz, 13353 Berlin, Germany. Institute for Bioinformatics, Department of Mathematics and Computer Science, Freie Universität Berlin, Takustrasse, 14195 Berlin, Germany. 


\section{Published online: 30 July 2015}

\section{References}

1. Dyment DA, Tetreault M, Beaulieu CL, Hartley T, Ferreira P, Chardon JW, et al. Whole-exome sequencing broadens the phenotypic spectrum of rare pediatric epilepsy: a retrospective study. Clin Genet. 2015;88:34-40.

2. Bamshad MJ, Shendure JA, Valle D, Hamosh A, Lupski JR, Gibbs RA, et al. The Centers for Mendelian Genomics: a new large-scale initiative to identify the genes underlying rare Mendelian conditions. Am J Med Genet A. 2012;158A:1523-5

3. St Hilaire C, Ziegler SG, Markello TC, Brusco A, Groden C, Gill F, et al. NT5E mutations and arterial calcifications. New Engl J Med. 2011;364:432-42.

4. Soden SE, Saunders CJ, Willig LK, Farrow EG, Smith LD, Petrikin JE, et al. Effectiveness of exome and genome sequencing guided by acuity of illness for diagnosis of neurodevelopmental disorders. Sci Transl Med. 2014;6:265ra168.

5. Zemojtel T, Kohler S, Mackenroth L, Jager M, Hecht J, Krawitz P, et al. Effective diagnosis of genetic disease by computational phenotype analysis of the disease-associated genome. Sci Transl Med. 2014;6:252ra123.

6. Yang Y, Muzny DM, Reid JG, Bainbridge MN, Willis A, Ward PA, et al. Clinical whole-exome sequencing for the diagnosis of mendelian disorders. New Engl J Med. 2013:369:1502-11.

7. Yang Y, Muzny DM, Xia F, Niu Z, Person R, Ding Y, et al. Molecular findings among patients referred for clinical whole-exome sequencing. JAMA. 2014;312:1870-9.

8. Lee H, Deignan JL, Dorrani N, Strom SP, Kantarci S, Quintero-Rivera F, et al. Clinical exome sequencing for genetic identification of rare Mendelian disorders. JAMA. 2014;312:1880-7.

9. Gilissen C, Hehir-Kwa JY, Thung DT, van de Vorst M, van Bon BW, Willemsen $\mathrm{MH}$, et al. Genome sequencing identifies major causes of severe intellectual disability. Nature. 2014;511:344-7.

10. van Zelst-Stams WA, Scheffer H, Veltman JA. Clinical exome sequencing in daily practice: 1,000 patients and beyond. Genome Med. 2014;6:2.

11. Boycott KM, Vanstone MR, Bulman DE, MacKenzie AE. Rare-disease genetics in the era of next-generation sequencing: discovery to translation. Nat Rev Genet. 2013;14:681-91.

12. Genomics England: First patients diagnosed through the 100,000 Genomes Project. http://www.genomicsengland.co.uk/first-patients-diagnosedthrough-the-100000-genomes-project/. Accessed 25 June 2015.

13. Rabbani B, Tekin M, Mahdieh N. The promise of whole-exome sequencing in medical genetics. J Hum Genet. 2014;59:5-15.

14. Mamanova L, Coffey AJ, Scott CE, Kozarewa I, Turner EH, Kumar A, et al. Targetenrichment strategies for next-generation sequencing. Nat Methods. 2010;7:111-8.

15. de Ligt J, Willemsen MH, van Bon BW, Kleefstra T, Yntema HG, Kroes T, et al. Diagnostic exome sequencing in persons with severe intellectual disability. New Engl J Med. 2012;367:1921-9.

16. Moreau Y, Tranchevent LC. Computational tools for prioritizing candidate genes: boosting disease gene discovery. Nat Rev Genet. 2012;13:523-36.

17. Robinson PN, Krawitz P, Mundlos S. Strategies for exome and genome sequence data analysis in disease-gene discovery projects. Clin Genet. 2011;80:127-32.

18. Gilissen $\mathrm{C}$, Hoischen A, Brunner HG, Veltman JA. Disease gene identification strategies for exome sequencing. Eur J Hum Genet. 2012;20:490-7.

19. Smith KR, Bromhead CJ, Hildebrand MS, Shearer AE, Lockhart PJ, Najmabadi $\mathrm{H}$, et al. Reducing the exome search space for mendelian diseases using genetic linkage analysis of exome genotypes. Genome Biol. 2011;12:R85.

20. Smedley D, Kohler S, Czeschik JC, Amberger J, Bocchini C, Hamosh A, et al. Walking the interactome for candidate prioritization in exome sequencing studies of Mendelian diseases. Bioinformatics. 2014;30:3215-22.

21. Jäger M, Wang K, Bauer S, Smedley D, Krawitz P, Robinson PN. Jannovar: a java library for exome annotation. Hum Mutat. 2014;35:548-55.

22. Wang K, Li M, Hakonarson H. ANNOVAR: functional annotation of genetic variants from high-throughput sequencing data. Nucleic Acids Res. 2010;38:e164.

23. Abecasis GR, Altshuler D, Auton A, Brooks LD, Durbin RM, Gibbs RA, et al. A map of human genome variation from population-scale sequencing. Nature. 2010;467:1061-73.

24. Sim NL, Kumar P, Hu J, Henikoff S, Schneider G, Ng PC. SIFT web server: predicting effects of amino acid substitutions on proteins. Nucleic Acids Res. 2012:40:W452-7.

25. Adzhubei IA, Schmidt S, Peshkin L, Ramensky VE, Gerasimova A, Bork P, et al. A method and server for predicting damaging missense mutations. Nat Methods. 2010;7:248-9.
26. Cooper GM, Stone EA, Asimenos G, Green ED, Batzoglou S, Sidow A. Distribution and intensity of constraint in mammalian genomic sequence. Genome Res. 2005;15:901-13.

27. Kircher M, Witten DM, Jain P, O'Roak BJ, Cooper GM, Shendure J. A general framework for estimating the relative pathogenicity of human genetic variants. Nat Genet. 2014;46:310-5.

28. McLaren W, Pritchard B, Rios D, Chen Y, Flicek P, Cunningham F. Deriving the consequences of genomic variants with the Ensembl API and SNP Effect Predictor. Bioinformatics. 2010;26:2069-70.

29. Exome Variant Server. NHLBI Exome Sequencing Project (ESP). http://evs.gs.washington.edu/EVS/. Accessed 25 June 2015.

30. ExAC Browser (Beta). Exome Aggregation Consortium (ExAC). http://exac.broadinstitute.org. Accessed 25 June 2015.

31. Robinson PN. Computational phenotype analysis in human medicine. In: Hancock J, editor. Phenomics. Boca Raton: CRC Press; 2014. p. 8-23.

32. Köhler S, Doelken SC, Mungall CJ, Bauer S, Firth HV, Bailleul-Forestier I, et al. The Human Phenotype Ontology project: linking molecular biology and disease through phenotype data. Nucleic Acids Res. 2014;42:D966-74.

33. Robinson PN, Webber C. Phenotype ontologies and cross-species analysis for translational research. PLOS Genet. 2014;10:e1004268.

34. Bauer S, Kohler S, Schulz MH, Robinson PN. Bayesian ontology querying for accurate and noise-tolerant semantic searches. Bioinformatics. 2012;28:2502-8.

35. Doelken SC, Kohler S, Mungall CJ, Gkoutos GV, Ruef BJ, Smith C, et al. Phenotypic overlap in the contribution of individual genes to CNV pathogenicity revealed by cross-species computational analysis of singlegene mutations in humans, mice and zebrafish. Dis Model Mech. 2013:6:358-72.

36. Schulz MH, Kohler S, Bauer S, Robinson PN. Exact score distribution computation for ontological similarity searches. BMC Bioinformatics. 2011;12:441.

37. Köhler S, Schulz MH, Krawitz P, Bauer S, Dolken S, Ott CE, et al. Clinical diagnostics in human genetics with semantic similarity searches in ontologies. Am J Hum Genet. 2009;85:457-64.

38. Sifrim A, Popovic D, Tranchevent LC, Ardeshirdavani A, Sakai R, Konings P, et al. eXtasy: variant prioritization by genomic data fusion. Nat Methods. 2013;10:1083-4.

39. Aerts S, Lambrechts D, Maity S, Van Loo P, Coessens B, De Smet F, et al. Gene prioritization through genomic data fusion. Nat Biotechnol. 2006:24:537-44.

40. eXtasy 0.1- Variant Prioritization by Genomic data Fusion. http://extasy.esat. kuleuven.be. Accessed 25 June 2015.

41. Singleton MV, Guthery SL, Voelkerding KV, Chen K, Kennedy B, Margraf RL, et al. Phevor combines multiple biomedical ontologies for accurate identification of disease-causing alleles in single individuals and small nuclear families. Am J Hum Genet. 2014;94:599-610.

42. Yandell $M$, Huff $C, H u H$, Singleton M, Moore B, Xing J, et al. A probabilistic disease-gene finder for personal genomes. Genome Res. 2011;21:1529-42.

43. Eppig JT, Blake JA, Bult CJ, Kadin JA, Richardson JE. The Mouse Genome Database (MGD): facilitating mouse as a model for human biology and disease. Nucleic Acids Res. 2015;43:D726-36.

44. Smith CL, Goldsmith CA, Eppig JT. The Mammalian Phenotype Ontology as a tool for annotating, analyzing and comparing phenotypic information. Genome Biol. 2005;6:R7.

45. Kibbe WA, Arze C, Felix V, Mitraka E, Bolton E, Fu G, et al. Disease Ontology 2015 update: an expanded and updated database of human diseases for linking biomedical knowledge through disease data. Nucleic Acids Res. 2015:43:D1071-8.

46. Gene Ontology Consortium. Gene Ontology Consortium: going forward. Nucleic Acids Res. 2015:43:D1049-56.

47. Amberger JS, Bocchini CA, Schiettecatte F, Scott AF, Hamosh A. OMIM.org: Online Mendelian Inheritance in Man (OMIM(R)), an online catalog of human genes and genetic disorders. Nucleic Acids Res. 2015;43:D789-98.

48. Phevor: Phenotype Driven Variant Ontological Re-ranking tool http://weatherby.genetics.utah.edu/cgi-bin/Phevor/PhevorWeb.html. Accessed 25 June 2015.

49. Javed A, Agrawal S, Ng PC. Phen-Gen: combining phenotype and genotype to analyze rare disorders. Nat Methods. 2014;11:935-7.

50. Robinson PN, Kohler S, Oellrich A, Wang K, Mungall CJ, Lewis SE, et al. Improved exome prioritization of disease genes through cross-species phenotype comparison. Genome Res. 2014;24:340-8. 
51. Smedley D, Oellrich A, Kohler S, Ruef B, Sanger Mouse P, Westerfield M, et al. PhenoDigm: analyzing curated annotations to associate animal models with human diseases. Database (Oxford). 2013;2013:bat025.

52. PhenIX. Charité-Universitätsmedizin Berlin. http://compbio.charite.de/PhenIX/. Accessed 25 June 2015.

53. Weedon MN, Cebola I, Patch AM, Flanagan SE, De Franco E, Caswell R, et al. Recessive mutations in a distal PTF1A enhancer cause isolated pancreatic agenesis. Nat Genet. 2014:46:61-4.

54. Oellrich A, Koehler S, Washington N, Sanger Mouse Genetic Program, Mungall C, Lewis $\mathrm{S}$, et al. The influence of disease categories on gene candidate predictions from model organism phenotypes. J Biomed Semantics. 2014;5:S4.

55. Masino AJ, Dechene ET, Dulik MC, Wilkens A, Spinner NB, Krantz ID, et al. Clinical phenotype-based gene prioritization: an initial study using semantic similarity and the human phenotype ontology. BMC Bioinformatics. 2014;15:248.

56. Grubb SC, Bult CJ, Bogue MA. Mouse phenome database. Nucleic Acids Res. 2014:42:D825-34.

57. Serikawa T, Mashimo T, Takizawa A, Okajima R, Maedomari N, Kumafuji K, et al. National BioResource Project-Rat and related activities. Exp Anim. 2009:58:333-41.

58. Mashimo T, Voigt B, Kuramoto T, Serikawa T. Rat Phenome Project: the untapped potential of existing rat strains. J Appl Physiol. 2005;98:371-9.

59. Cheng KC, Xin X, Clark DP, La Riviere P. Whole-animal imaging, gene function, and the zebrafish Phenome Project. Curr Opin Genet Dev. 2011;21:620-9.

60. The Monarch Initiative. http://monarchinitiave.org. Accessed 25 June 2015.

61. Fokkema IF, Taschner PE, Schaafsma GC, Celli J, Laros JF, den Dunnen JT. LOVD v. 2.0: the next generation in gene variant databases. Hum Mutat. 2011;32:557-63.

62. National Research Council (US) Committee on A Framework for Developing a New Taxonomy of Disease. Toward precision medicine. Building a knowledge network for biomedical research and a new taxonomy of disease. Washington (DC): National Academies Press (US); 2011.

63. Beaulieu CL, Majewski J, Schwartzentruber J, Samuels ME, Fernandez BA Bernier FP, et al. FORGE Canada Consortium: outcomes of a 2-year national rare-disease gene-discovery project. Am J Hum Genet. 2014;94:809-17.

64. Bamshad MJ, Ng SB, Bigham AW, Tabor HK, Emond MJ, Nickerson DA, et al Exome sequencing as a tool for Mendelian disease gene discovery. Nat Rev Genet. 2011;12:745-55.

65. Gahl WA, Markello TC, Toro C, Fajardo KF, Sincan M, Gill F, et al. The National Institutes of Health Undiagnosed Diseases Program: insights into rare diseases. Genet Med. 2012;14:51-9.

66. Bragin E, Chatzimichali EA, Wright CF, Hurles ME, Firth HV, Bevan AP, et al. DECIPHER: database for the interpretation of phenotype-linked plausibly pathogenic sequence and copy-number variation. Nucleic Acids Res. 2014;42:D993-1000.

67. Wright CF, Fitzgerald TW, Jones WD, Clayton S, McRae JF, van Kogelenberg $\mathrm{M}$, et al. Genetic diagnosis of developmental disorders in the DDD study: a scalable analysis of genome-wide research data. Lancet. 2015;385:1305-14.

68. Kumar P, Henikoff S, Ng PC. Predicting the effects of coding nonsynonymous variants on protein function using the SIFT algorithm. Nat Protoc. 2009:4:1073-81.

69. Schwarz JM, Cooper DN, Schuelke M, Seelow D. MutationTaster2: mutation prediction for the deep-sequencing age. Nat Methods. 2014;11:361-2.

70. Lopes MC, Joyce C, Ritchie GR, John SL, Cunningham F, Asimit J, et al. A combined functional annotation score for non-synonymous variants. Hum Hered. 2012;73:47-51. 